\title{
Luminescence of water and ice as a new detection channel for neutrino telescopes
}

\author{
Anna Pollmann ${ }^{a, *}$ \\ ${ }^{a}$ University of Wuppertal, \\ Gaußstr. 20, Wuppertal, Germany \\ E-mail: anna.pollmann@uni-wuppertal.de
}

Natural water and ice are currently used as optical detection media in large scale neutrino telescopes, such as IceCube, KM3NeT/ANTARES and GVD. When charged particles, such as those produced by high energy neutrino interactions, pass through ice or water at relativistic speeds they induce Cherenkov light emission. These photons are recorded by the optical sensors of neutrino telescopes. However, slower moving particles, including potential exotic matter such as Magnetic Monopoles or Q-balls, cannot be recorded using this detection channel.

New kinds of signatures can be detected by using light emission from luminescence in water or ice. This light is induced by highly ionizing particles passing through a medium and exciting the surrounding matter. This detection channel enables searches for exotic particles which are too slow to induce Cherenkov light emission and currently cannot be probed by neutrino telescopes. Luminescence light is possibly highly dependent on the ice quality, solubles, and temperature. Therefore a comprehensive study is demanded.

To utilize luminescence as a new detection channel in neutrino telescopes, laboratory measurements using water and ice as well as an in-situ measurement in Antarctic ice were performed. The experiments as well the measurement results will be presented covering light yields, spectra and decay times. The impact on searches for new physics with neutrino telescopes will be discussed.

$3^{\text {th }}$ International Cosmic Ray Conference (ICRC 2021)

July 12 th - 23rd, 2021

Online - Berlin, Germany

\footnotetext{
${ }^{*}$ Presenter
} 


\section{Introduction}

The largest detectors for elementary particles on Earth are neutrino telescopes. Their huge volume of up to a cubic kilometer is optimal to search for rare event signatures such as high energetic neutrinos or particles which are predicted beyond the standard model of particle physics. The current telescopes, KM3NeT, Baikal GVD, IceCube, and Hyper-Kamiokande, use water and ice as detection medium. When a particle from space or the Earth's atmosphere interacts with the medium, charged particles could be produced. If the speed of these particles exceeds the speed of light in the medium, Cherenkov light is emitted. However, neutral or slow particles cannot be detected using Cherenkov light.

In order to use the large volume of neutrino detectors for searches for neutral or slow exotic particles, a different detection channel than Cherenkov light is required. Luminescence light emission does not have the restrictions on charge and speed which motivates a thorough measurements of its properties to investigate if it can be used as a second detection channel at neutrino telescopes.

When ionizing radiation is passing through matter, it excites atoms, molecules or the lattice to a higher electronic state. These excited states decay with an exponential function over time emitting photons isotropically, the luminescence light. The excess energy can also be dissipated via non-radiative processes. Quenching, which is the reduction of emitted photons, is correlated with the electrical charge of the incident particle as well as the presence of impurities in the medium acting as quenchers.

Luminescence of a medium is characterized by the light yield and quenching, the decay time, as well as the emission spectrum. In order to use luminescence of water and ice as a detection channel for elementary particles, the light yield has to be sufficiently high so that the considered detector can record the particle signature. The decay times need to be sufficiently short to enable the reconstruction of the signature. Also the spectrum should fit the detector's specifications. Whether these requirements are met by luminescence of water and ice, is investigated in this work.

\section{Measurement setups}

The properties of luminescence of ice at conditions given at the telescopes has been measured in laboratory as well as in-situ at South Pole. In this chapter the experimental setups are described.

\subsection{Laboratory setup}

In the laboratory, ultra-pure water ${ }^{1}$ was used. Also, bubble free ice sheets were frozen from this water under clean conditions. Five ice samples were used each for the presented measurements. Luminescence was induced with two different radioactive sources in order to investigate the quenching effect: an ${ }^{241} \mathrm{Am}$ source emitting mainly $\alpha$-particles, and a customized, titanium covered ${ }^{36} \mathrm{Cl}$ source emitting mainly $\beta$-particles. The light, in form of single photons, was then measured with a photomultiplier ${ }^{2}$ (PMT). The geometry of the optical setup was measured and the transmission was calculated. Background effects were investigated with the source being covered by a light tight

\footnotetext{
${ }^{1} \mathrm{HLPC}$ grade: total organic carbon (TOC) $<5 \mathrm{ppb}$, conductivity $>18 \mathrm{M} \Omega$

${ }^{2}$ Hamatsu R1924P, 1 inch, with magnetic shielding, driven by the high voltage module iseg THQ, read out by a PicoScope 6404B.
} 

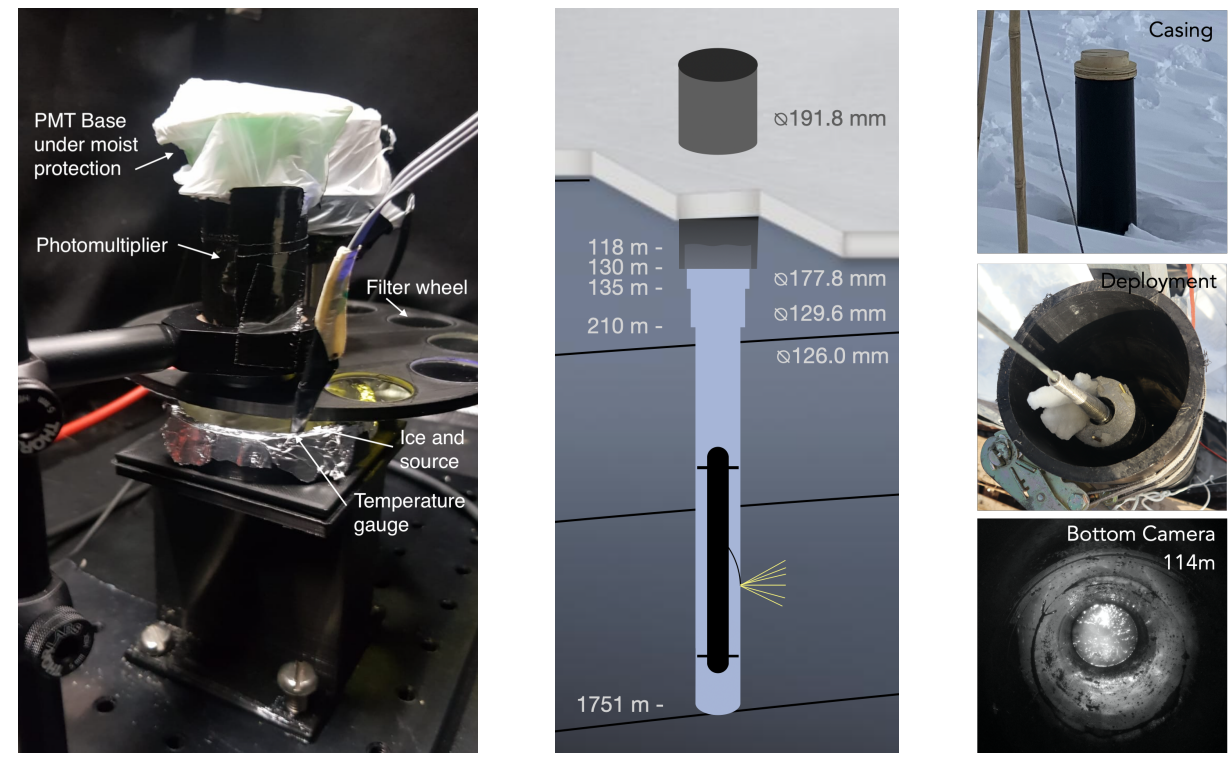

Figure 1: Left: Setup in a refrigerator to measure the spectrum of luminescence light. Middle: Bore hole map of the SPICEcore hole. Right: Pictures of the hole: the casing sticking out of the snow surface, the deployment of a logger into the casing, the surface of the liquid at around $114 \mathrm{~m}$ photographed with an on-board camera.

lid, see previous report in Ref. [1]. The raw data was cleaned from obvious electronic noise, PMT dark noise as well as temperature dependent effects. In order to record a rough spectrum, long-pass filters ${ }^{3}$ were moved in between the sample and the PMT automatically with a wheel using a motor and a decoder suitable for cold temperatures, see Figure 5.

\subsection{In-situ setup}

The in-situ measurement was conducted at South Pole where the SPICEcore borehole [2] provides access to the ice of the IceCube neutrino observatory. The ice ${ }^{4}$ which could be probed had an effective overlap with the IceCube depths of about $350 \mathrm{~m}$. The borehole is about $1.7 \mathrm{~km}$ deep with a diameter of $12.6 \mathrm{~cm}$ below $210 \mathrm{~m}$, see Figure 2. It is filled with a liquid ${ }^{5}$ to provide sufficient pressure to keep it from collapsing.

The original setup from the laboratory measurement needs to be placed into a pressure vessel ${ }^{6}$ to withstand up to $170 \mathrm{bar}$ in the hole, see Figure 2 and Ref. [4]. The $\beta$-source is attached on a spring outside of the vessel. With the help of magnets, which are rotated by a motor, it can be pressed onto the ice wall at measurement positions where the electrons are emitted into the ice. The induced Cherenkov and luminescence light is back-scattered in the ice to some degree. If it falls onto the mirror ${ }^{7}$ inside the vessel, it is reflected onto the photomultiplier. A filter holder, providing three slots, can be driven into the light path in order to record a rough spectrum.

\footnotetext{
${ }^{3}$ Edmund optics High Performance OD 4.0 Longpass Filters

${ }^{4}$ TOC ca. 7 ppb [3]

${ }^{5}$ Estisol-140

${ }^{6}$ Nautilus quartz glass, $1.3 \mathrm{~m}$ long, $9.2 \mathrm{~cm}$ outer diameter

${ }^{7}$ Thorlabs off-axis parabolic mirror, UV-enhanced, 2 inch
} 

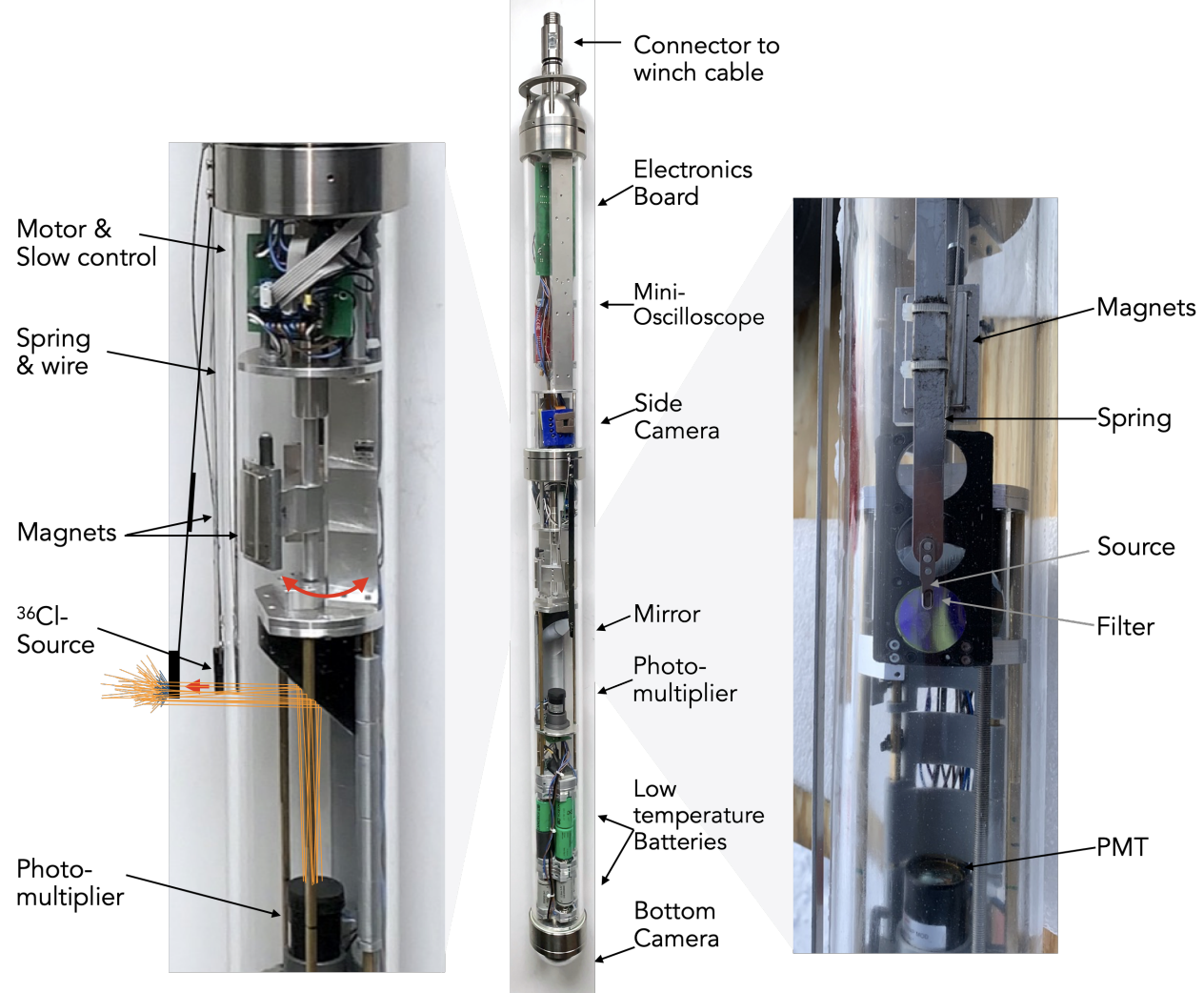

Figure 2: Middle: Photograph of the luminescence logger with a zoom into the important mechanics and optics (left) as well as onto the filter optics (right).

The PMT waveforms are recorded by a mini-oscilloscope ${ }^{8}$ which also functions as the main on-board computer. It provides communication ${ }^{9}$ for live-read-out and control of the logger from a surface PC. Power for the computers is provided over the winch cable ${ }^{10}$. To drive the motors, eight low temperature batteries ${ }^{11}$ are placed in the logger. Additional four batteries drive LEDs for two on-board IR-cameras ${ }^{12}$. Environmental sensors were used to record the temperature at several locations inside the logger as well as the orientation and acceleration of the logger. The depth was automatically read-out from the winch.

Measurements were conducted in December 2018 and 2019. In the first austral summer season the light yield and decay kinetics were measured at three different depth. In the second season the light yield was measured in about 30 different depths and the spectrum was taken in three different depths which required three different deployments to measure with all of the eight filters given a 3-slot filter holder. The logger is capable to carry other experiments instead of the luminescence measurement, see Ref. [5].

Optical and electrical properties of the logger as well as the source properties were measured

\footnotetext{
${ }^{8}$ RedPitaya

${ }^{9}$ Serial RS232 over 2 conductors with a customized half-duplex protocol

${ }^{10} \mathrm{DC}+/-48 \mathrm{~V}+$ grounding via the cable shield

${ }^{11} \mathrm{CR} 26500$

${ }^{12}$ Raspberry pi zero plus Raspberry IR camera with 800 nm LEDs
} 


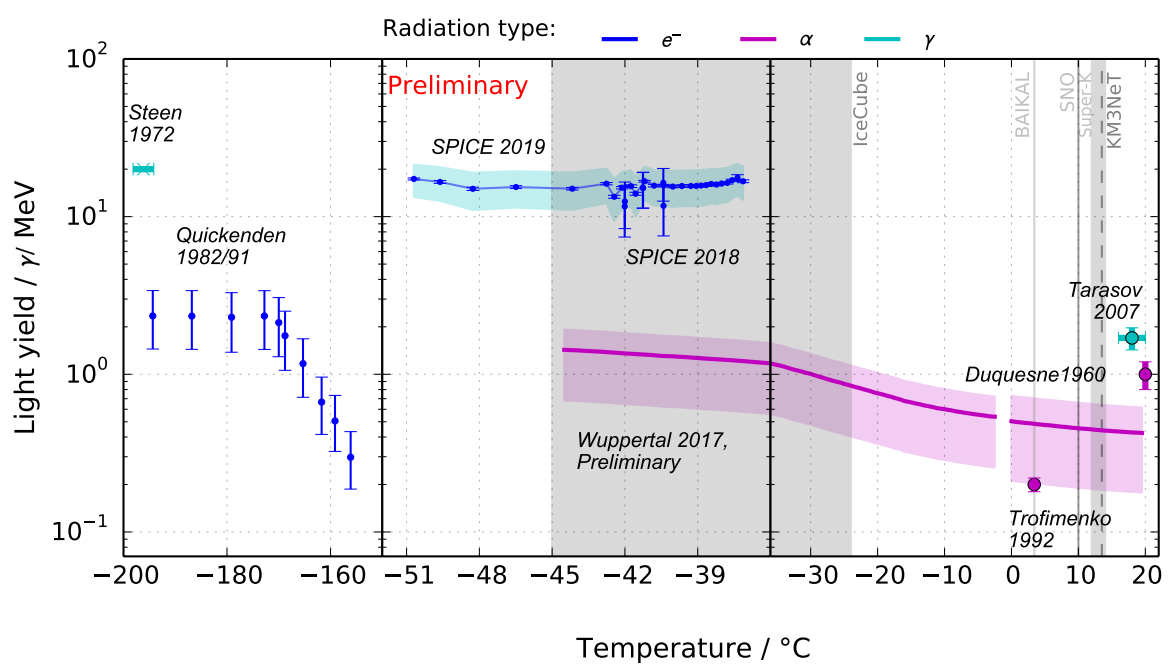

Figure 3: Summary of this and all previous measurements of the light yield of luminescence of water and ice in dependence of Temperature. Temperatures given at different neutrino telescopes are shown in gray bands. The type of irradiation inducing luminescence light is denoted by the color of measurement points / lines. For this work, shaded areas denote systematic uncertainties and bars denote statistical uncertainties. [REFs]

in laboratory. The source emission was modeled ${ }^{13}$ and the light emission (Cherenkov light and luminescence in the bore hole liquid) and propagation was simulated with a custom ray tracing, taking local scattering and absorption coefficients into account. The raw data was cleaned from obvious electronic noise and PMT dark noise as well as temperature dependent effects.

\section{Measurement results}

The light yield of luminescence light, shown in Fig. 3 was obtained from the recorded PMT single photon rates with data treatment as described in the previous section. The laboratory measurement, taken with the $\alpha$-source, shows a strong temperature dependence.

The in-situ measurement, taken with the $\beta$-source provided an order of magnitude higher values for the light yield ${ }^{14}$. This could be due to quenching by $\alpha$-particles or the different ice quality.

Measurements of the ice solubles were compared with the pattern observed in the rate of the in-situ measurement and a correlation with the $\delta^{18} \mathrm{O}$ ratio in the dissolved $\mathrm{O}_{2}$ molecule [8] was found (Pearson coefficient -0.51). Since the ice at South Pole originally consists of snow compactified by subsequent layers of snow, it contains a significant amount of ambient air which could contribute to the measured luminescence light yield.

The spectrum of luminescence was measured with the long-pass filters providing an integrated spectrum. It was cleaned and corrected as described above. In the laboratory measurement with the $\beta$-source the distinct feature of the spectrum is a dip at $375 \mathrm{~nm}$. This dip is consistent over all temperatures. However, in the measurement with the $\alpha$-source, this dip is at a higher wavelength of

\footnotetext{
${ }^{13}$ GEANT4

${ }^{14}$ The depth of the in-situ measurement relates inversely to the temperature.
} 


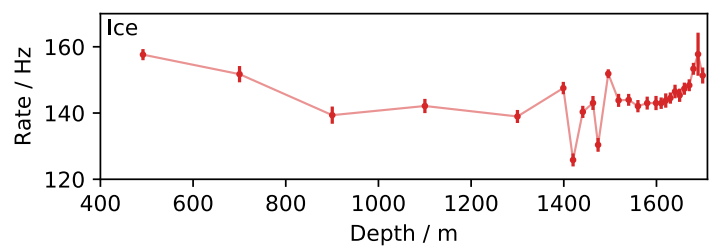

Figure 4: Cleaned rate of the in-situ measurement of luminescence which has been corrected for temperature effects and Cherenkov and bore hole liquid contributions.
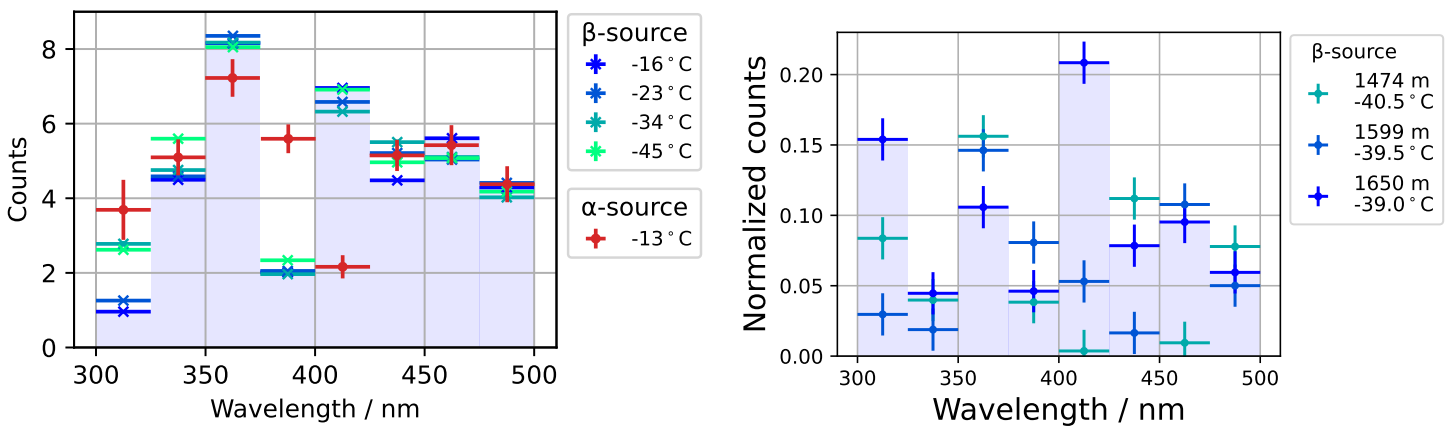

Figure 5: Left: The spectrum of luminescence measured in laboratory at different temperatures of ice using both sources. Right: The spectrum measured in-situ at three different depths in three deployments. In each deployment the rate was recorded with three different filters (or no filter) applied.

$400 \mathrm{~nm}$. This indicates a quenching effect which would change or move the emitted photon energy of the first peak to higher wavelengths.

The prominent dip at $375 \mathrm{~nm}$ is also present in the in-situ measurement. However, the rate at $400 \mathrm{~nm}$ is inconsistent for different depths or temperatures. A second dip is observed at $325 \mathrm{~nm}$ which is consistent in all measurements. The variation at $400 \mathrm{~nm}$ originates from the ice quality in which solubles might quench or enhance certain photon energy emissions.

The decay kinetics was measured in-situ in the first deployment season, reported in Ref. [4]. Four different decay times could be identified which are consistent over the the different depths. They range from $2.5 \mathrm{~ns}$ up to $56 \mu \mathrm{s}$. The decay time in dependence of the wavelength range will be analyzed with the data recorded in the second field season.

\section{Applications}

The results, presented above, were tested by simulating particles which emit luminescence light with these properties. As an example the signature of a charged Q-ball is shown in Fig. 6. Q-balls are predicted in super-symmetric theories to be coherent states of squarks, sleptons and the Higgs-field [9]. They are interesting candidates of dark matter and could be charged or neutral. The latter would interact with matter via the KKST nucleon-decay process. However, the former could not be searched for at neutrino telescopes with conventional methods.

The event display in Fig. 6 shows a clear track despite the sparse instrumentation of the IceCube detector. Due to the slow speed of the particle, most PMTs see noise during the event duration 

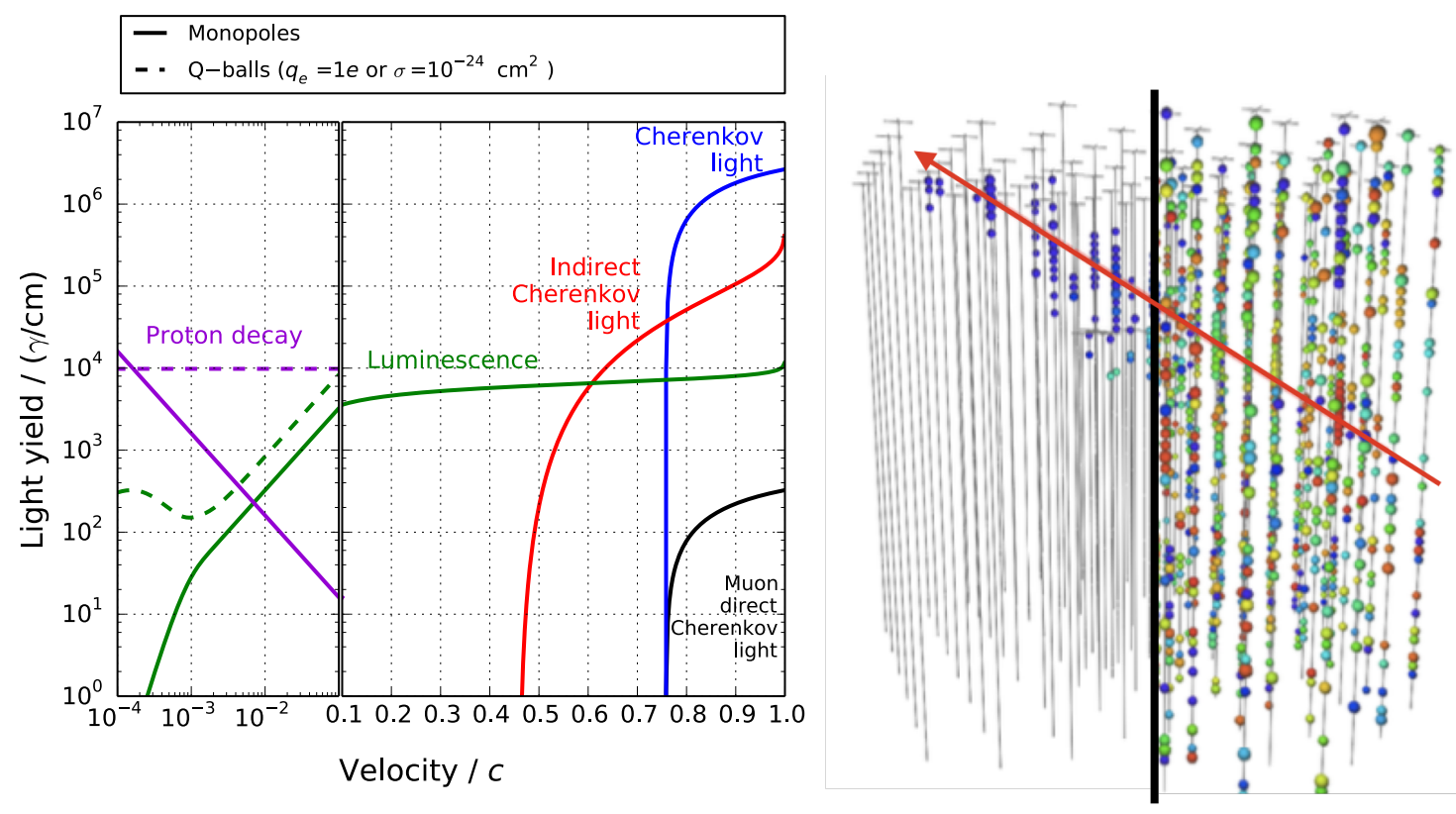

Figure 6: Left: Light yield of different emissions for magnetic monopoles carrying one Dirac charge and Q-balls with a charge of $10^{20}$ in comparison to a bare muon passing through ice. Right: Event signature of a Q-ball simulated with luminescence emission. The Q-ball has a speed of $10^{-3} c$, a charge of $10^{20}$, and an electric charge of $137 e$. (original pictures of this composed picture are taken from Ref. [6]).

which hides the track in noise which can be removed in the analysis [6].

Another particle, which can be searched for using luminescence, is a magnetic monopole. These are predicted by Grand-Unified theories and others to be particles carrying at least one isolated magnetic charge [10]. Similar to the Q-balls these Big Bang relics are assumed to be very massive, however monopoles can be accelerated to relativistic speeds by inter-galactic magnetic fields. At low and high speeds detection channels are the model dependent catalysis of nucleon decay via the Rubakov-Callan effect and (in-) direct Cherenkov light. However, at intermediate speeds as well as low speeds no (model in-dependent) light emission was known before.

The first search ever using luminescence light as detection channel for Magnetic Monopoles passing through the IceCube detector at low relativistic speeds has been finalised [7]. The sensitivity of this analysis exceeds previous limits in this region by approximately two orders of magnitude. The exclusion limit therefore catches up with the IceCube limits at other speed ranges which are currently World leading.

Luminescence light also contributes to the total light emission of Standard Model particles, especially the interaction products of ultra high energy neutrinos. This has consequences for the energy, signature topology or particle ID reconstruction because taking luminescence into account could improve accuracy of these by uo to $10 \%$.

\section{Summary and outlook}

In this work various measurents of luminescence of water and ice in laboratory and in-situ are presented and compared. A clear indication of the influence of the radiation type as well as the ice 
properties can be seen.

The properties proved sufficient to detect signatures of exotic particles with high sensitivity which could not be reached with other, smaller detectors than neutrino telescopes.

Future work focuses on investigating the procceses leading to luminescene emission of ice which could be inferred by the presented data.

Acknowledgements: The author would like to thank the SPICEcore collaboration for providing the borehole, the US Ice Drilling Program, the Antarctic Support Contractor and the National Science Foundation for providing the equipment to perform the described measurement and for their support at South Pole.

\section{References}

[1] A.Pollmann, S.Pieper, PoS ICRC2017 1060

[2] SPICEcore Collaboration, Annals of Glaciology 55 (2014) 68

[3] S. Preunkert, Environ. Sci. Technol. 45, 2 (2011) 673-678

[4] A.Pollmann, PoS ICRC20119 983

[5] IceCube Collaboration, PoS ICRC2021 1047 (these proceedings)

[6] S.Pieper, University of Wuppertal (2020) (Master Thesis)

[7] IceCube Collaboration, PoS ICRC2021 534 (these proceedings)

[8] E.J.Steig et al., USAP Data Center (2018) doi: https://doi.org/10.15784/601114

[9] A.Kusenko, M.E.Shaposhnikov, Phys. Lett. B418 (1998) 46-54

[10] A.Rajantie. Contemporary Physics 53, 3 (2012) 195-211 